\title{
The Kinematic Analysis of the Screw Conveyor Vibration Exciter
}

\author{
Dmitriy Kobylyansky ${ }^{1}$, Viacheslav Gogolin ${ }^{1}$ \\ ${ }^{1}$ T.F. Gorbachev Kuzbass State Technical University, Underground Mining Department, 650000 \\ Kemerovo, 28 Vesennya st., the Russian Federation
}

\begin{abstract}
The article presents a method of calculating the parameters of the screw conveyor vibration exciter. The calculation scheme of the new design of the vibration exciter, which allows to determine the parameters of the vibrations excited by this device taking into account the structural novelty and the specifics of the vibration exciter, in particular, in the screw reloader is also given. A system of equations describing the harmonic oscillations of the auger shaft axis, including the amplitudes of horizontal and vertical oscillations, is presented. As a result, a system of parametric equations describing the external and internal envelope line is obtained. When solving the system of equations, the equations of the boundary curves of internal and external rotating bodies are derived. The equations allow to calculate the trajectory of any point by its given polar coordinates. Examples of rotating bodies for different types of displacements of the triangle center in the calculation scheme, which are described by harmonic oscillations, are shown. The dependences of the body shape on the direction of oscillations along the coordinate axes are illustrated. It is shown that the transition from horizontal to vertical oscillations leads to a loss of symmetry of the body. The amplitude and frequency of oscillations also have a significant impact on the shape of the body. The forms of the rotating bodies, giving the possibility of combining the rotation and oscillation when driving on rollers are determined. The problem is generalized to an arbitrary number of regularly arranged rollers. The given technique gives the principal opportunity to create designs of vibrators that combine vibrations with rotation on rollers.
\end{abstract}

\section{Introduction}

A new design of the vibration exciter of the screw conveyor $[1,2]$ has been proposed, which requires the development of a method for calculating the parameters of oscillations excited by this device, taking into account the structural novelty and the specifics of the work, in particular, in the screw reloader.

Let us determine the simplest form of a rotating body-cam (close to the oval) according to the calculation scheme (Fig.1). form:

We set the equation of harmonic oscillations of the screw shaft axis in the following 


$$
\left\{\begin{array}{c}
f(t)=a \cdot \sin 3 t, \\
g(t)=b \cdot \cos 3 t-b,
\end{array}\right.
$$

where: $\mathrm{a}, \mathrm{b}$ are the amplitudes of the horizontal and vertical axis oscillation; $f(t)$ displacement on the axis $\mathrm{O}_{1} \mathrm{X}_{1} ; g(t)$ - displacement on the axis $\mathrm{O}_{1} \mathrm{Y}_{1} ; t$ - time.

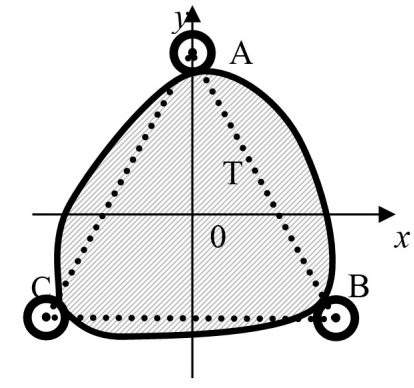

a)

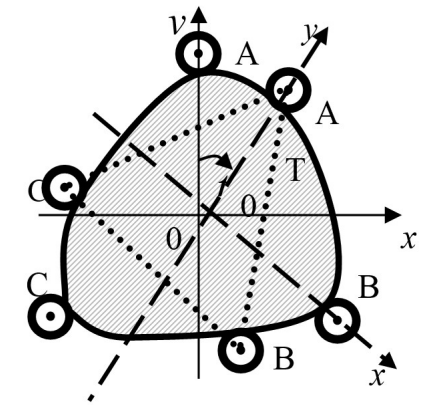

b)

Fig.1. The calculation scheme.

In the work [3] the system of parametric equations of the envelope line in the coordinate system $\mathrm{X}_{1} \mathrm{O}_{1} \mathrm{Y}_{1}$ is obtained:

$$
\left\{\begin{array}{l}
x_{1}=R \sin t+f(t) \pm r \cdot \frac{R \sin t-g^{\prime}(t)}{\sqrt{\left[R \sin t-g^{\prime}(t)\right]^{2}+\left[R \cos t+f^{\prime}(t)\right]^{2}}}, \\
y_{1}=R \cos t+g(t) \pm r \cdot \frac{R \cos t+f^{\prime}(t)}{\sqrt{\left[R \sin t-g^{\prime}(t)\right]^{2}+\left[R \cos t+f^{\prime}(t)\right]^{2}}} .
\end{array}\right.
$$

The resulting system of equations (2) describes two envelope lines: with a plus sign the outer envelope of the rollers, with a minus sign - the inner envelope one $[4,5]$.

After substitution (1) in (2) we obtain:

$$
\left\{\begin{array}{l}
x_{1}=R \sin t+a \sin 3 t-r \cdot \frac{R \sin t+3 b \sin 3 t}{\sqrt{D_{1}}}, \\
y_{1}=R \cos t+b \cos 3 t-b-r \cdot \frac{R \cos t+f^{\prime}(t)}{\sqrt{D_{1}}},
\end{array}\right.
$$

where: $D_{1}=R^{2}+6 a R \cos t \cos 3 t+6 b R \sin t \sin 3 t+9 a^{2} \cos ^{2} 3 t+9 b^{2} \sin ^{2} 3 t$.

At the same values of amplitudes $\mathrm{a}=\mathrm{b}=\mathrm{h}$ the expression (4) takes the form:

$$
D_{1}=R^{2}+6 h R \cos 2 t+18 h^{2} .
$$

The equations (3) and (4) describe the shape of the body before its motion begins [6].

Let us move from the coordinate system associated with the body $\mathrm{X}_{1} \mathrm{O}_{1} \mathrm{Y}_{1}$, into the coordinate system XOY, associated with the triangle ABC (see Fig.1), and thus describe the movement of the body on rollers [7]. To do this, we move the body along the coordinate axes by $-\mathrm{f}(\varphi)$ horizontally, by $-\mathrm{g}(\varphi)$ vertically and turn the body by an angle $\varphi$ counterclockwise relative to the vertical axis. When the body is rotated by an angle $\varphi$, as a result of the transformation of equations (3) and (4), we obtain the following equations of the boundary curves of the inner and outer body in the coordinate system XOY: 


$$
\left\{\begin{array}{l}
x(\varphi)=\left[x_{1}-f(\varphi)\right] \cos \varphi-\left[y_{1}-g(\varphi)\right] \sin \varphi, \\
y(\varphi)=\left[x_{1}-f(\varphi)\right] \sin \varphi+\left[y_{1}-g(\varphi)\right] \cos \varphi,
\end{array}\right.
$$

The equation (6) allows us to describe the trajectory of any point according to its given polar coordinates $\mathrm{R}, \mathrm{t}$. To describe the trajectory of the instantaneous center of rotation of the body in the equation 6 , put $\mathrm{R}=0=\mathrm{t}$, which leads to a parametric equalization of this trajectory:

$$
\left\{\begin{array}{l}
x=-f(\varphi) \cos \varphi+g(\varphi) \sin \varphi, \\
y=-f(\varphi) \sin \varphi-g(\varphi) \cos \varphi .
\end{array}\right.
$$

The Fig.2 shows the examples of bodies (envelope curves) for different types of displacements of the triangle center, which are described by harmonic oscillations. Here and further all the dimensions are shown in relative units of radius $\mathrm{R}=1$. Curve 1 shows the inner envelope curve, curve 2 corresponds to the outer envelope or the body making the same movements with the external touch of the rollers $[8,9]$. 


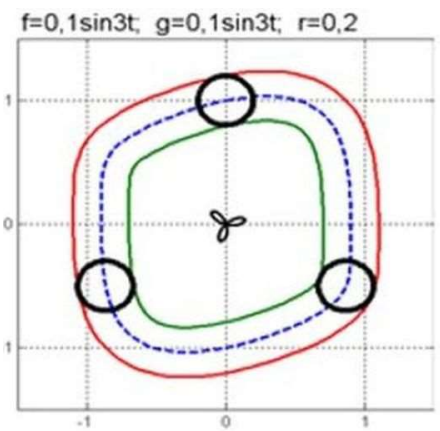

a)

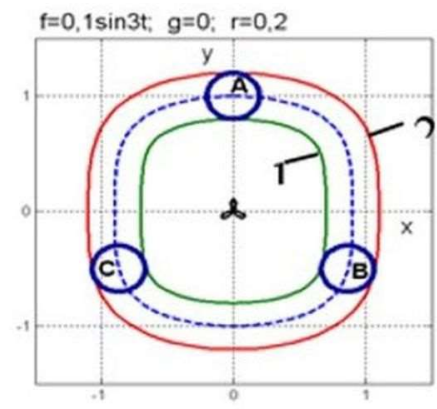

c)

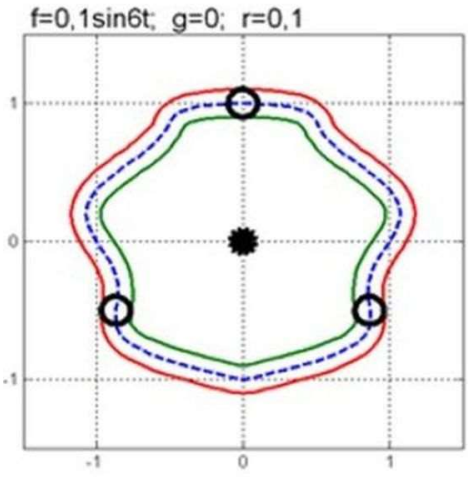

e)

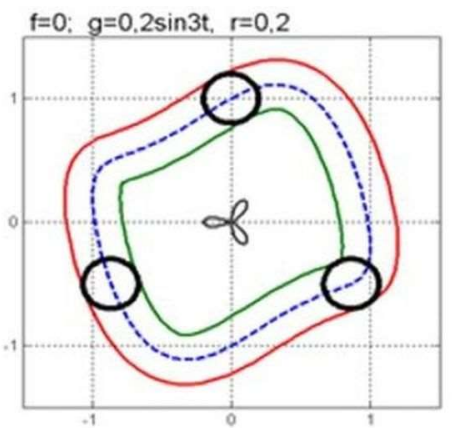

b)

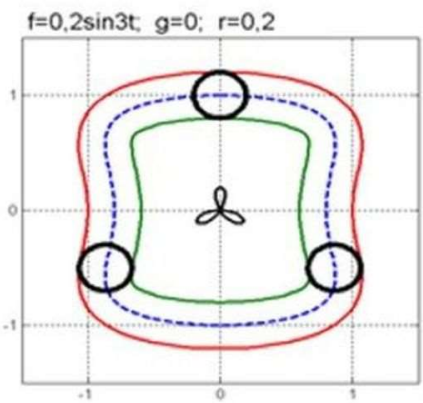

d)

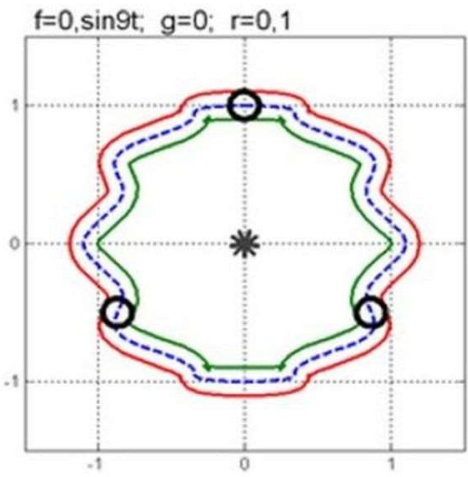

f)

Fig. 2. Forms of bodies and the trajectories of their rotation centers.

The dotted curve describes the limiting case - the boundary of the body for the rollers of zero radius or, in other words, the trajectory of the centers of the rollers when they are rolled as a whole on the outer 2 or inner envelope 1 . In the center of each of the individual figures the trajectory of the centers of the bodies rotation is shown. The determination of these trajectories will be mentioned hereinafter.

These examples illustrate the dependence of the body shape on the direction of oscillations along the coordinate axes: along the OX axis (Fig.2a, b, e, f), along the axis OY (Fig.2d) and on both axes (Fig.2c). Moreover, the transition from horizontal to vertical oscillations leads to a loss of the body symmetry. The amplitude and the frequency of 
oscillations also have a significant influence on the shape of the body. With an increase in the oscillation amplitude along the axis OX from 0.1 to 0.2 the body having an oval shape (Fig.2a), takes the form with convex and concave parts (Fig.2b). With an increase in the oscillation frequency determined by the angle of rotation multiplying factor $t$, the number of areas of convexity and concavity of the body increases (Fig.2b, e, f), and as it is seen in Fig.2e, at a high frequency of oscillations, nodal points may appear at the boundary of the inner body [10]. The remaining parameter that determines the shape of the body is the radius of the rollers, the effect of which is seen in Fig.2.

Fig. 3 shows the position of the internal and external bodies when moving on the rollers (see Fig.2b). In the center of each individual figure there is a part of the trajectory of the instantaneous center of rotation of the bodies when they rotate at a specified angle $\varphi$, constructed by the equation (7). This figure illustrates the possibility of combining the rotation of the bodies and their oscillations when moving on rollers. It should be noted that there is no other possibility of the body movement along three rollers of equal radius with centers at the vertices of an equilateral triangle, since the body in any position must have one degree of freedom to rotate around the instantaneous center [11]. In this case, the position of the instantaneous center of rotation should be consistent with the rotation of the body, as done above in (2), (7).

The considered problem is generalized to an arbitrary number of $n$ rollers regularly arranged so that their centers coincide with the vertices of a regular n-gon. Repeating the above mentioned for this case, we come to the same parametric equations (2), (7) with the following conditions on the values of the moving the instantaneous center of rotation functions instead of the conditions:

$$
\left\{\begin{array}{l}
f(0)=f(2 \pi / 3)=f(4 \pi / 3)=0, \\
g(0)=g(2 \pi / 3)=g(4 \pi / 3)=0 .
\end{array}\right.
$$

In this case we obtain:

$$
f(2 k \pi / n)=g(2 k \pi / n)=0, k=0, \ldots, n-1 .
$$

It should be noted that, for example, all the functions of the following form meet the requirements $(8)$ :

$$
H(t)=h_{0}+\sum_{m=1}^{N} h_{1 m}(t) \cdot \sin (3 m t)+h_{2 m}(t) \cdot \cos (3 m t) .
$$

The following condition must be met $h_{0}=-\sum_{m=1}^{N} h_{2 m}(0)$.

Functions of the form (10) describe a fairly wide class of curves, in particular, such as cycloids and trochoids, even when the multipliers of the sine and cosine are constant. When these multipliers are variables, it is sufficient to require their continuous differentiability to have functions $\mathrm{f}, \mathrm{g}$ of rotation center displacements corresponding to the mechanical meaning of the problem.

A sufficiently wide class of functions satisfying the condition (9) in this case instead of (10) will have the following form: 


$$
H(t)=h_{0}+\sum_{m=1}^{N} h_{1 m}(t) \cdot \sin (n m t)+h_{2 m}(t) \cdot \cos (n m t) .
$$

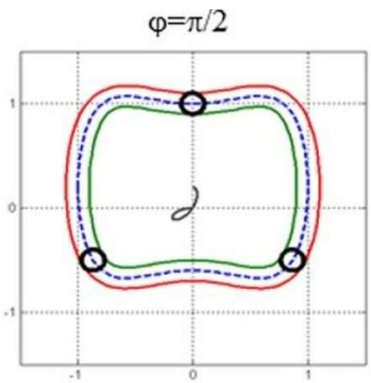

a)

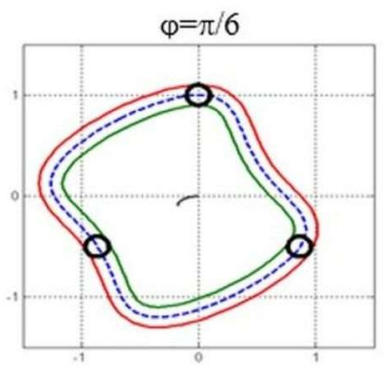

c)

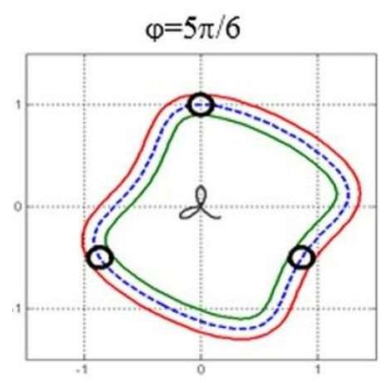

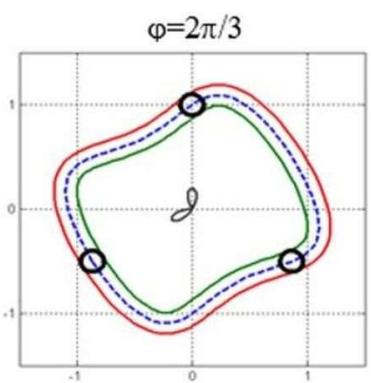

b)

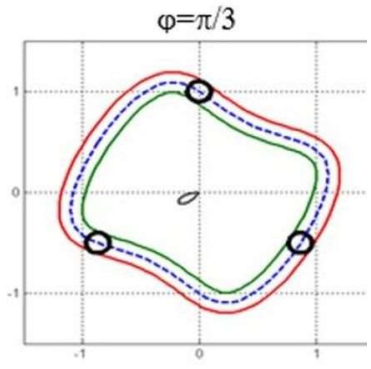

d)

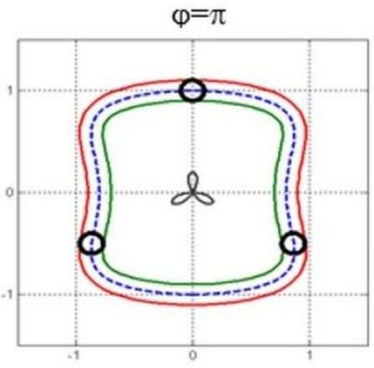

f)

Fig. 3. The stages of body movement on rollers.

The use of other, more complex types of functions does not cause difficulties, and was tested in the program developed by the authors in the MATLAB package.

The presented method of calculation of the bodies held by the rollers rotation and oscillation was designed in particular to calculate the reference nodes screw conveyors vibration exciters $[12,13]$

When designing the mechanisms the nodes of which have a limited number of degrees of freedom in the presence of roller connections, the question arises about the influence of the geometric parameters of the nodes and the kinematic characteristics of motion on their possible structural shape. Such mechanisms, in particular, include vibrators that combine vibrations with rotation. With the help of the calculation method given above, the principal possibility of creating designs of such vibrators has been established, and the variety of body forms combining vibrations with the rotation on rollers has been shown. 


\section{References}

1. H. Zareiforoush, M. H. Komarizadeh, M. R. Alizadeh, and M. Mosoomi, Journal of Agricultural science, 2:2, 147 (2010)

2. H. Zareiforoush, M. H. Komarizadeh, and M. R. Alizadeh, Nature and Science, 8:6, 66 (2010)

3. H. Zareiforoush, M. H. Komarizadeh, and M. R. Alizadeh, Research Journal of Applied Sciences, Engineering and Technology, 1:3, 132 (2009)

4. H. A. Araghi, M. Sadeghi, and A. Hemmat, International Agrophysics, 24:2, pp. 205 (2010)

5. T. K. Ray, Mechanical Handling of Materials (Asian Books Private, New Delhi, India, 2004)

6. W. Roberts, Powder Technology, 104:1, 56 (1999)

7. G. Hu, J. Chen, B. Jian, H. Wan, and L. Liu, International Conference on Mechanic Automation and Control Engineering, 5536244, 927 (2010)

8. J. W. Fernandez, P. W. Cleary, and W. McBride, Seventh International Conference on CFD in the Minerals and Process Industries CSIRO, 1, (2009)

9. W. Roberts, Bulk Solids, Chemical engineering, 122:2, 62, (2015)

10. S. Xiaoxia, M. Wenjun, and Y. Yuan, Advances in Mechanical Engineering, 9:7, 1687814017714984, (2017)

11. Y. Tian, P. Yuan, F. Yang, J. Gu, M. Chen, J. Tang, Q. Cheng, Applied Sciences, 8:7, 1038 (2018)

12. O. L. Lyashuk, O. R. Rogatynska, and D. L. Serilko, INMATEH: Agricultural Engineering, 45, 87, (2015)

13. B. M. Hevko, Yu. F. Pavel'chuk, Innovative solutions in Modern Science, 10:1, 1 (2017) 